\title{
RESULTS FROM THE HIPPARCOS MISSION ON STELLAR SEISMOLOGY
}

\author{
L. EYER AND M. GRENON \\ Geneva Observatory, CH-1290 Sauverny, Switzerland
}

\section{Introduction}

Photometric results from the HIPPARCOS mission are of interest to stellar seismology. HIPPARCOS provides a general description of the HR diagram in terms of stability, microvariability and variability. The satellite has detected systematically variables with amplitudes exceeding a threshold, strongly magnitude dependent but as small as a few mmag for bright stars. When the signal is periodic, periods and amplitudes are determined. Because of the peculiar time sampling of HIPPARCOS only first modes and associated amplitudes are generally obtained. This information is particularly relevant to seismology in the case of pulsating stars as $\delta$ Scuti stars, slowly pulsating $\mathrm{B}$ stars and $\beta$ Cephei stars.

\section{The Main Mission HIPPARCOS}

The main mission produced more than 13 million photometric measurements in the $H p$ wide band for 118204 stars. That is an average of 110 measurements per star over a time span of 3.3 years. The observed stars were issued from a selection, which consisted of a survey of around 53000 stars complete to the magnitude $H p<7.9+1.1 \sin (b)$ for stars earlier than G5 and $H p<7.5+1.1 \sin (b)$ for stars later than G5, where $b$ is the galactic latitude, and a set of 65000 fainter stars selected for their astrophysical or astrometrical interest.

\subsection{THE TIME SAMPLING AND THE PHOTOMETRIC PRECISION}

The two ingredients, time sampling and precision, are critical in order to know what kind of variability can be detected. With HIPPARCOS, these properties are rather heterogeneous. The basic time sampling can be seen through the example in Fig. 1 . The smallest intervals occur in the successive sequence: $20-108-20$-etc... minutes. The compact groups of such transits are often separated by around one month. The detection limit for period is mainly 40 minutes, however lower periods can be detected in principle (cf. Eyer and Bartholdi 1998). The photometric precision depends strongly on the magnitude. The data are heteroscedastic (individual transits have different precisions). We give in Table 1 the mean behaviour of the precision versus the magnitude $H p$ (cf. Eyer and Grenon 1997). 


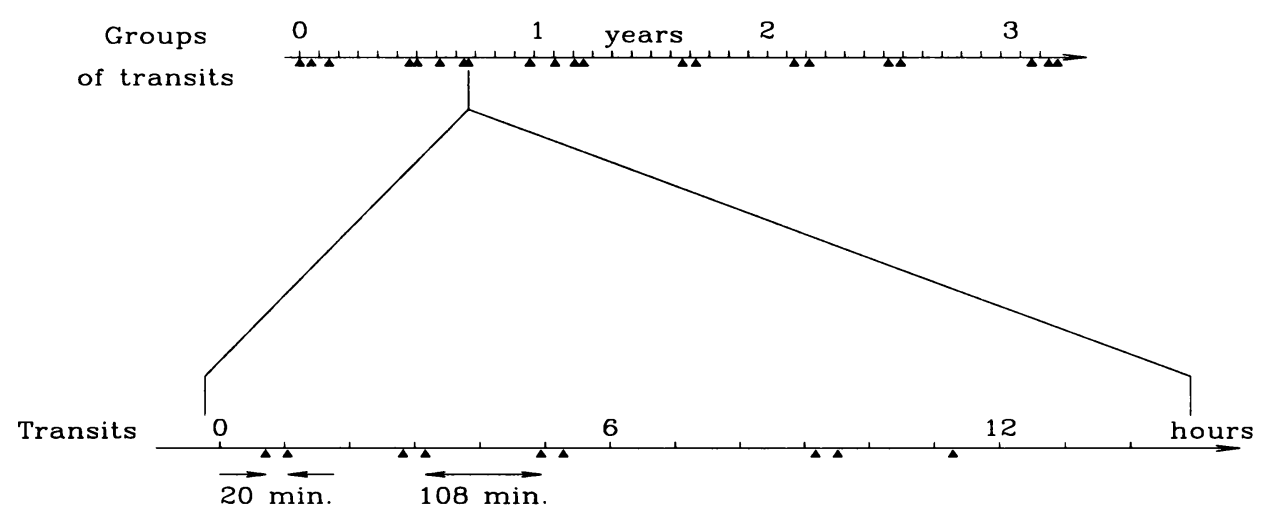

Figure 1. A typical time sampling (example HIP 059862).

TABLE 1. Precision on $H p$ magnitude of individual transits as function of $H p$

\begin{tabular}{l|llllllll}
\hline$H p$ & 3 & 5 & 7 & 8 & 9 & 10 & 11 & 12 \\
\hline Precision (mmag) & 3 & 5 & 7 & 12 & 17 & 25 & 36 & 55 \\
\hline
\end{tabular}

\subsection{THE RESULTS}

A general analysis, done in a collaboration between the Geneva Observatory and the Royal Greenwich Observatory, resulted in the publication of two Catalogues about variables stars and three associated Atlases (cf. van Leeuwen et al. 1997 and Grenon et al. 1997). The catalogue of Periodic Variables contains information about period, epoch, extrema and their associated precisions for 2712 stars. The Catalogue of Unsolved variables gives information about the amplitude of variability for 5442 stars. No periodic solutions were found for these stars. 4086 variables were new discoveries and 1955 stars received names by Samus (1998).

\section{Focus on seismology}

\subsection{NEW VARIABLES}

The HIPPARCOS all-sky monitoring was free from biases in favour of particular spectral types, or time sampling as from the Earth; it allowed a systematic detection of variables and hence many new discoveries.

In Table 2 we display, for few variable types of seismological interest, the total number of stars known before HIPPARCOS, and the number discovered by HIPPARCOS with an amplitude and a period.

A study of the $\beta$ Cephei stars and SPBs was done by Waelkens et al. (1997), who multiplied nearly by 10 the number of known SPBs. A thorough discussion of these stars is presented by Aerts et al. (these proceedings). 
TABLE 2. Low amplitude pulsators observed by HIPPARCOS

\begin{tabular}{lll}
\hline Variable type & Known (suspected) & New \\
\hline$\beta$ Cep & $59(80)$ & 4 \\
SPB & 11 & $72-103$ \\
$\delta$ Scuti & $271(19)$ & 35 \\
$\gamma$ Dor & $11(17)$ & $\sim 20$ \\
roAp & 29 & 0 \\
\hline
\end{tabular}

For cooler stars, especially $\gamma$ Dor stars and $\delta$ Scuti stars, a similar study will be done in order to characterize their pulsation modes and evolutionary status. A rough estimate of the number of new suspected $\gamma$ Dor stars is around 20 according to Eyer (1998).

In Fig. 2, we put together all HIPPARCOS stars with $\sigma_{H p}<0.2 \mathrm{mag}$ (dots), and data from literature (open circles or crosses; squares and triangles for $\gamma$ Dor stars and suspected $\gamma$ Dor stars, circles for $\delta$ Scuti stars, and crosses for roAp). Full circles are the data from HIPPARCOS for different period intervals (different grey levels). There is a clump around $M_{v} \simeq 2.8$ and $B-V \simeq 0.3$ of unevolved main sequence stars with higher periods intermediate between those of $\delta$ Scuti stars and $\gamma$ Dor stars $(0.25<$ $\mathrm{P}<0.5$ ). These new variables are at the red edge of the instability strip and of the Böhm-Vitense gap. On main sequence, the blue edge of the instability strip does not extend bluewards of $(\mathrm{B}-\mathrm{V})=0.14$.

Rapidly oscillating Ap stars are too rapidly pulsating and have too small amplitudes to be detected by HIPPARCOS. In addition, their periods are not necessarily stable over 3 years.

\subsection{DETECTION OF MULTIPLE PERIODS}

From simulations (using sinusoidal signals and the CLEAN method), we learn that several periods may be often recovered from the data. However, the possibility to find several periods depends not only on the relative intensities of the amplitudes and of the noise level but also on the value and separation of the periods (for a given star). Furthermore, it depends on the peculiar sampling of each star. Therefore it is, according to our experience, risky to deduce multiperiodic behaviour from the HIPPARCOS data alone, because stars like $\delta$ Scuti stars are not always stable over a period of 3 years (example GX Peg), and that the spectrum can lead to wrong conclusions because of the aliasing problem. Period confirmation and a-posteriori studies can be, however, fruitful (e.g. the two main periods in 1 Mon can be recovered). This can lead to the conclusion of the long term stability of the pulsation characteristics.

\section{Short time scale variations and further studies}

Using an estimator of the scatter of the pairwise differences in different but short time intervals (cf. Eyer and Genton 1998), we detected very short-term variables (or 


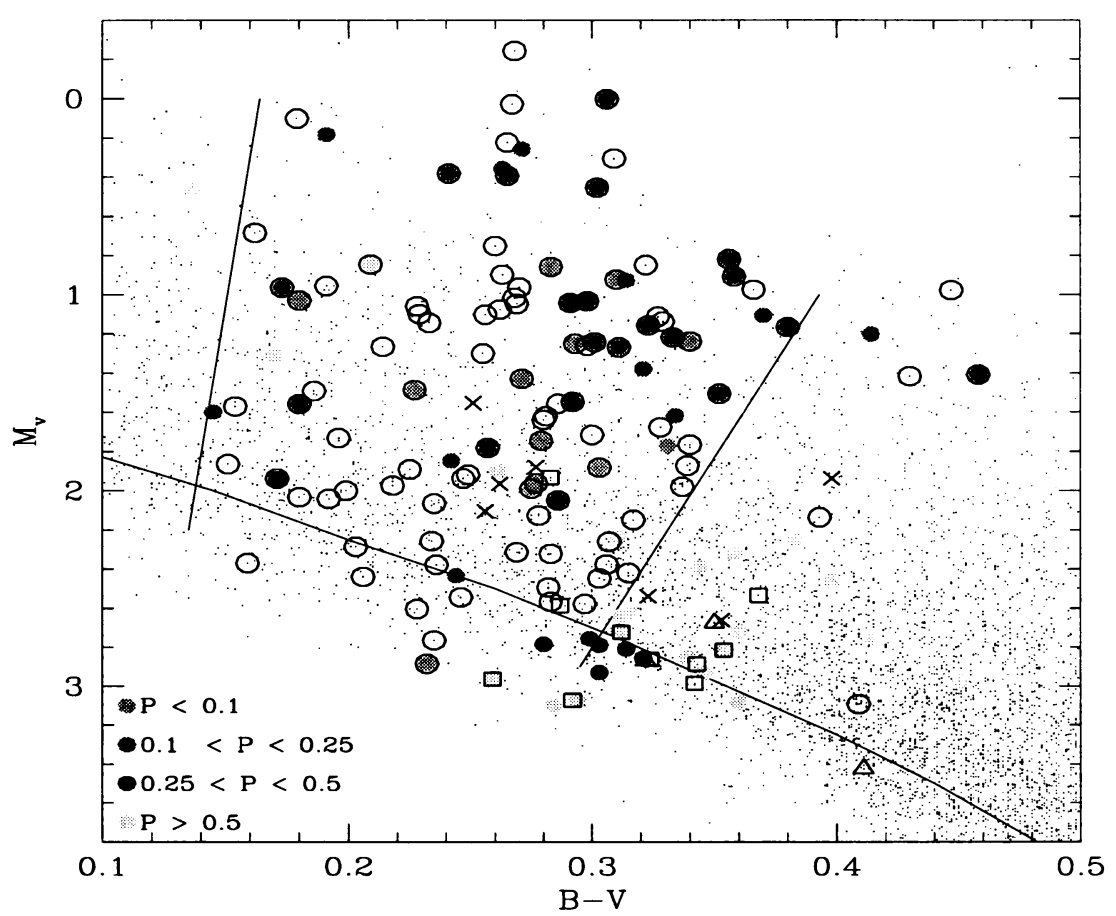

Figure 2. HR-diagram for stars near the instability strip. Filled symbols : HIPPARCOS variables.

light curves with locally large derivatives). We found three variables, HIP 071119 , HIP 044025, which are $\delta$ Scuti stars and HIP 029055 which is the large amplitude and known RR Lyrae VW Dor; these stars are not in the catalogue of periodic variables. Thus, the catalogue of unsolved variables will reveal many more interesting cases as expected.

\section{References}

Eyer, L., and Grenon, M. (1997) Photometric Variability in the HR-diagram, ESA SP-420 Eyer, L., and Genton, M. (1998) in preparation Eyer, L., and Bartholdi, M. (1998), A\&A, in preparation

Eyer, L. (1998) Hipparcos et les étoiles variables, PhD Thesis, Observatoire de Genève Garcia, J.R. et al. (1995) $A \& A S, 109,201$

Grenon, M. et al. (1997) ESA SP-1200, Volume 12

Krisciunas, K. and Handler, G. (1995) IBVS 4195

van Leeuwen, F. et al. (1997) ESA SP-1200, Volume 11

Samus, N. et al. (1998) $I B V S$, in preparation

Sterken, C. and Jerzykiewicz, M. (1993) Space Science Reviews, 62, 95

Waelkens, C. et al. (1997), A\&A submitted 